\title{
Sex differences in the association of phospholipids with components of the metabolic syndrome in young adults
}

\author{
Sebastian Rauschert ${ }^{1}$, Olaf Uhl' ${ }^{1}$, Berthold Koletzko ${ }^{1 *}$, Trevor A. Mori ${ }^{2}$, Lawrence J. Beilin², Wendy H. Oddy ${ }^{3,4}$
} and Christian Hellmuth ${ }^{1}$

\begin{abstract}
Background: There are differences in the prevalence and severity of diseases between males, females not taking hormonal contraceptives (non-HC females) and females taking hormonal contraceptives (HC females). The aim of this study was to identify sex-specific differences in the metabolome and its relation to components of the metabolic syndrome in a young adult population.

Methods: The subjects analysed are from the 20-year follow-up of the Western Australian Pregnancy Cohort (Raine) Study. Two hundred fifteen plasma metabolites were analysed in 1021 fasted plasma samples by a targeted liquid chromatography coupled to tandem mass spectrometry (LC-MS/MS) metabolomics approach. Principal component analysis between males $(n=550)$, non-HC females $(n=199)$ and HC females $(n=269)$ was applied. Regression analysis with a sex $\times$ metabolite concentration interaction was performed on components of the MetS, namely waist circumference, systolic blood pressure, and plasma $\mathrm{HDL}-\mathrm{C}$, triglycerides and glucose concentration, as outcome to select the significant metabolites of the interaction. Those selected metabolites were used as predictors in a sex group stratified analysis to compare the different $\beta$ coefficients and therefore the sex group-dependent associations.

Results: Principal component analysis between males, non-HC females, and HC females showed a general discriminating trend between males and $\mathrm{HC}$ females. One hundred twenty-seven metabolites were significantly different between males and non-HC females, whereas 97 differed between non-HC females and $\mathrm{HC}$ females. Males and non-HC females mainly differed in sphingomyelin, lyso-phosphatidylcholine, acyl-carnitine and amino acid species, whilst non-HC females and HC females mainly differed in phosphatidylcholine, lyso-phosphatidylcholine and acyl-carnitine concentrations. Forty-one metabolites (phosphatidylcholines, sphingomyelines, lysophosphatidylcholine) were significantly differently associated with the MetS factors in the different groups.

Conclusions: We have shown clear differences between plasma metabolite concentrations in males, and HC or non-HC females, especially in lyso-phosphatidylcholine, sphingomyelin and phosphatidylcholine, which have been shown to associate with obesity in other studies. The association of these metabolites differed between sexes with components of the metabolic syndrome, which means that development of diseases like obesity and diabetes may differ between the sexes. Our findings highlight the importance of considering sex differences when conducting a metabolomics study and the need to account for the effect of HC usage in females in future studies.
\end{abstract}

Keyword: Metabolic syndrome, Sex differences, Metabolomics, Raine study, Sphingolipids, Hormonal contraceptives

\footnotetext{
* Correspondence: office.koletzko@med.Imu.de

'Ludwig-Maximilian Universität München, Dr. von Hauner Children's Hospital,

University of Munich Medical Center, Lindwurmstr. 4, D-80337 München,

Germany

Full list of author information is available at the end of the article
} International License (http://creativecommons.org/licenses/by/4.0/), which permits unrestricted use, distribution, and reproduction in any medium, provided you give appropriate credit to the original author(s) and the source, provide a link to the Creative Commons license, and indicate if changes were made. The Creative Commons Public Domain Dedication waiver (http://creativecommons.org/publicdomain/zero/1.0/) applies to the data made available in this article, unless otherwise stated. 


\section{Background}

There is increasing evidence from epidemiological studies that sex and oral contraceptive use in females modulate the development and the severity of cardiovascular disease [1-4]. For example, men are more likely to develop coronary heart diseases than women, especially women prior to menopause, but the risk of developing stroke and heart failure is higher in women [1]. Similarly, more females than males are overweight and obese in developing countries, whereas the opposite is found in developed countries [5]. Since males and females differ in the development and severity of obesity and insulin resistance, it is likely that differences in their metabolism, as a result of genetic effects and environmentally induced changes, may play an important role.

Analysis of the metabolome of males and females could provide insights into the sex-related differences of progression and severity of cardiovascular diseases. Most of the metabolomics studies to date have not stratified analyses according to sex, most likely due to their small sample size. Additionally, to our knowledge there are no studies that have examined sex differences in metabolomics and associated these with components of the metabolic syndrome (MetS). In particular, the characterisation of hormonal contraceptive usage in females with regards to its potential effect on metabolomic changes is highly underrepresented in current research, although a large number of women have been using them since their development in the 1960s [3].

This topic is of high relevance in the approach of implementing sex-specific interventional strategies and biomarker tests in future clinical settings. If biomarkers are different between the sexes, the relevance of those general biomarkers is questionable and also critical in terms of false diagnosis.

The aim of this study was to examine the metabolome of males and females at 20 years of age, including females either using $(\mathrm{HC})$ or not using hormonal contraceptives (non-HC), taking part in the Western Australian Pregnancy Cohort (Raine) Study. The study related potential differences in the metabolome to a number of risk factors underlying the MetS [6].

\section{Methods}

Details on the Raine study have been reported previously [7, 8]. Briefly, from 1989 to 1991, 2900 pregnant women were enrolled in this prospective longitudinal cohort study with the purpose to examine the effects of prenatal ultrasound imaging on the offspring [8]. Two thousand eight hundred sixty-eight live births were evaluated and followed serially to 20 years of age. The present study used cross-sectional data from the 20-year follow-up of the cohort, which occurred between March 2010 and April 2012 and included 87\% of the active participants.
Ethics approval at the 20 year follow-up was obtained from the University of Western Australia Human Research Ethics Committee. Informed and written consent was obtained from participants.

Serum insulin, glucose, lipids and liver function tests were analysed using standardised protocols in the PathWest Laboratory at Royal Perth Hospital, Perth, Western Australia.

MetS components were the ones established by the International Diabetes Federation, including central obesity, raised systolic blood pressure (sysBP), fasting raised triacylglycerol (TG), glucose, and reduced high density lipoprotein cholesterol (HDL-C) [6]. HC use in females was derived from a questionnaire and based on current use of the oral contraceptive pill, implant, injection or any intrauterine $\mathrm{HC}$-device and defined as a binary variable yes/no.

\section{Metabolomics measurements}

Polar lipids (acyl-carnitines (acyl-Carn), diacyl-phosphatid ylcholines (PCaa), acyl-alkyl-phosphatidylcholines (PCae), sphingomyelines (SM), lyso-phosphatidylcholines (LPC/ LPCa), alkyl-linked lyso-phosphatidylcholines (LPCe)), non-esterified fatty acids (NEFA) and amino acids (AA) were measured as previously reported [7, 9, 10].

The Additional file 1 provides a detailed description of the metabolomics methodology. Briefly, proteins of plasma were precipitated by adding methanol including internal standards. After centrifugation, the supernatant was used for analyses by liquid chromatography (1200, Agilent) coupled to triple quadrupole mass spectrometry (4000QTRAP, Sciex). Metabolites were quantified by comparison to external standards as $\mu \mathrm{mol} / \mathrm{L}$.

\section{Potential confounding factors}

Physical activity was assessed using the short form of the International Physical Activity Questionnaire and asked whether individuals performed more than $10 \mathrm{~min}$ of moderate or vigorous physical activity, and time spent walking or sitting in the last 7 days. The number of days, hours and minutes was reported. We created a categorical variable with "less than once a week", "1 to 3 times" and " 4 or more times" more than 10 min of physical activity per week.

Sedentary behaviour assessment was based on hours spent in front of a screen, including TV watching, playing videogames, socializing and non-socializing activities on the Internet.

Smoking cigarettes and drinking alcohol were used as binary variables. Smoking was assessed by asking if the participant was currently smoking. Alcohol consumption was based on any alcohol consumption in the last 7 days. 


\section{Ethnicity was applied as a dichotomous variable of Caucasian versus non-Caucasian.}

Diet was based on dietary patterns as reported by Ambrosini et al. and included three variables: a healthy dietary pattern, a western dietary pattern and a dietary misreporting variable of food intake to account for over, under, or plausible dietary reporting [11].

\section{Statistics}

The software $\mathrm{R}$ ( $\mathrm{R}$ Project for Statistical Computing, http://www.r-project.org/, Version 3.0.2) was used for all statistical analyses. To determine if the residuals were normally distributed, we checked the diagnostic plots of all the models. The $R$ function crPlots from the car package in $R$ [12] was used to check if outliers influence the linearity. We also assessed the boxplots for all variables used, leading to outlier exclusion. Together with our sufficiently large sample size (Central Limit Theorem), the model assumptions were met. Z-scores for each metabolite within each batch have been calculated to account for batch variation. A batch was defined as 81 samples together with standards and 6 quality control samples. All samples were measured in 15 batches.

We defined a three-level sex variable with the categories males, non-HC females and $\mathrm{HC}$ females.

The analysis was divided in three parts. First of all, we aimed at general sex differences.

\section{General sex differences: principal component analysis}

To determine whether the variance in the metabolomics data could be explained in part by the sex categories, we applied principal component analysis (PCA), which is used to find components that explain most of the variance in the data. The components explaining most of the variance (component 1 and component 2) were plotted against each other to see if they showed discriminating clusters for the sex categories. To assess which metabolite groups drive that discrimination, the loadings of the metabolite classes were depicted as arrows in the same plot.

\section{Sex differences in the associations with the components of the MetS: analysis of variance}

In the second analysis, we aimed to extract those metabolites that were significantly associated with at least one of the components of the MetS, depending on the sex variable.

Therefore, the metabolites that were significantly different between the three levels of the sex variable in association with the five continuous factors of the MetS needed to be identified. This pre-selection of metabolites was performed by applying a sex and metabolite interaction variable into analysis of variance (Anova) models with one of the five MetS indicators per model as outcome adjusted for the above-mentioned confounders. A significant interaction meant that the association between the metabolite and the single MetS factor was different depending on at least one of the three sex categories. To indicate which sex groups differed, we performed regression analysis to compare the three group differences after the anova.

\section{Regression models stratified by sex group}

The third analysis aimed to determine if the effect size of the metabolite in association with the single component of the MetS was significantly different between the three sex categories.

The metabolites that were significant in the interaction with at least one of the factors associated with the MetS were used to analyse if the effect sizes are significantly different between the sex categories. Therefore, we stratified the data into a male and non- $\mathrm{HC}$ and $\mathrm{HC}$ female subset. In all analyses, one of the five factors associated with the MetS was the outcome per model and the identified metabolites the predictor, adjusted for confounding variables.

The resulting standardized $\beta$ coefficients were compared between the three sex categories. Significance for the difference of the $\beta$ coefficients was tested by applying a regression model with dummy variables (male and non-HC female, HC female, each: yes (1), no $(0))$, to determine if they were significantly different to the reference category. This difference can be interpreted as the difference in the association between the metabolite and the outcome, if the respective sex category is added. It is a way to perform a significance test for the difference of $\beta$ coefficients in different categories (for an overview of the analysis strategy, see Fig. 1).

To determine if the metabolites that showed up significantly in the stratified groups were of any significance in the general, unstratified population, we performed analyses with the whole data set, where a single one of the five MetS indicators was the outcome and metabolite concentration was the predictor, adjusted for the confounders stated above (Additional file 1: Table S1).

We used false discovery rate as described by Benjamini and Hochberg to account for multiple testing for the $p$ values [13]. This was performed using the p.adjust function of the $\mathrm{R}$ core package. The confidence intervals were corrected for multiple testing using the formula $1-\frac{\# \text { of selected metabolites } \times 0.05}{\# \text { of all metabolites }}$, according to Benjamini and Yekutieli [14]. The correction procedure for multiple testing was applied using the number of metabolites. This approach was used, as the main aim of this study was hypothesis generation, and the Bonferroni correction is very restrictive in this regards. 


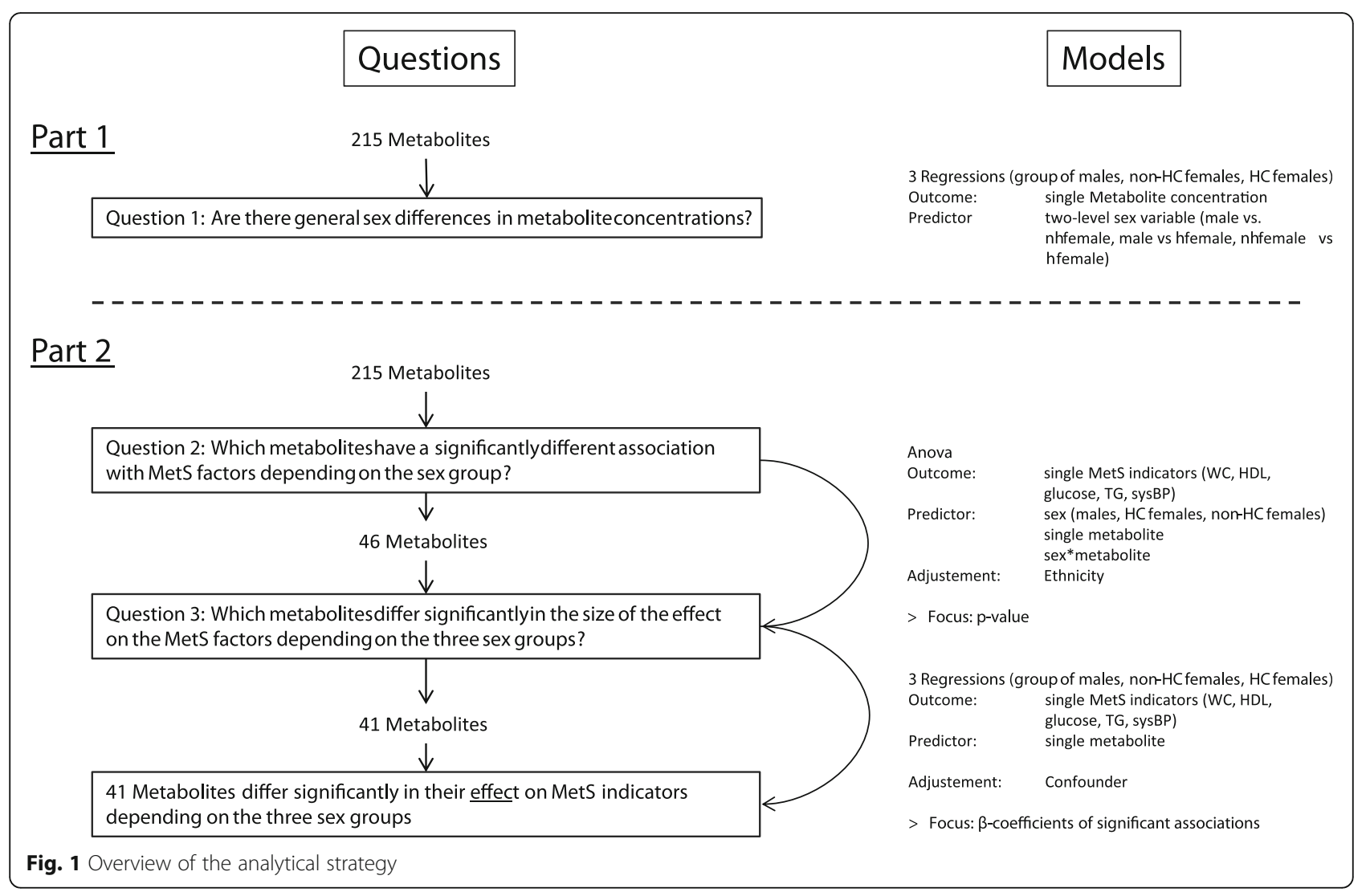

\section{Results}

The study characteristics of the participants are shown in Table 1. Results for all models and metabolites can be found in the Additional file 1: Table S1 which shows that there were significant differences in the concentrations of HDL-C, glucose and triglycerides, sysBP and waist circumference (WC) between males and non-HC and $\mathrm{HC}$ females.

\section{General sex differences: principal component analysis}

PCA (Figure Fig. 2) showed a clustering effect between males and $\mathrm{HC}$ females, predominantly due to differences in SM, PC and LPC.

In the regression, there were 127 metabolites significantly different between males and non- $\mathrm{HC}$ females and 97 metabolites that differed significantly between $\mathrm{HC}$ and non-HC females.

Males and $\mathrm{HC}$ females, however, significantly differed in 161 metabolites (Additional file 1: Table S2Table S4, Table 2).

\section{Sex differences in the associations with components of the MetS: analysis of variance}

In the Anova, we found that 46 metabolites showed a sex interaction with at least one component of the MetS (Additional file 1: Table S5, group differences
Additional file 1: Table S5). These 46 metabolites were included in the stratified analysis to examine the standardized $\beta$ values. All of the 46 metabolites were significantly associated with the indicators of the MetS in the unstratified analysis of the general Raine study population.

Those metabolites were acyl-Carn C3:0, NEFA C24:5, NEFA C26:1, PCaa C32:3, PCaa C34:1, PCaa C34:2, PCaa C34:3, PCaa C36:0, PCaa C36:1, PCaa C36:2, PCaa C36:3, PCaa C36:4, PCaa C38:0, PCaa C38:3, PCaa C38:4, PCaa C38:5, PCaa C38:6 , PCaa C40:6, PCaa C40:4, PCaa C40:5, PCae C36:1, PCae C38:0, PCae C40:0, PCae C40:6, SM C36:0, SM C38:1, SM C38:2, SM C40:3, SM C40:4, SM C40:5, SM C41:0, SM C42:4, SM C42:6, SM C44:6, LPCa C16:0, LPCa C16:1, LPCa C18:0, LPCa C18:1, LPCa C18:3, LPCa C20:3, LPCa C20:4, LPCa C20:5, LPCa C22:5, LPCa C22:6, LPCe C18:0, and LPCe C18:1 (for sex group differences: Table 3 , for $p$ values, $\beta$-coefficients and confidence interval: Additional file 1: Table S6).

In regression models stratified by sex group, we found that 41 of these metabolites had significantly different between-group $\beta$ coefficients, whereas acyl-Carn C3:0, PCaa C34:2, PCaa C40:4, SMa C38:2 and NEFA C26:1 showed no significant difference (Additional file 1: Table S5). None of the metabolites were significantly different between the sex categories for all MetS factors. 
Table 1 Study participant characteristics stratified by sex and hormonal contraceptive (HC) use

\begin{tabular}{|c|c|c|c|c|}
\hline & Females not taking $\mathrm{HC}$ & Females taking $\mathrm{HC}$ & Males & $p$ value \\
\hline$n(\%)$ & $199(19.55)$ & $269(26.42)$ & $550(54.03)$ & \\
\hline $\begin{array}{l}\text { Age (years; mean } \pm s d) \text { Age (years; } \\
\text { mean } \pm s d) \text { Age (years; mean } \pm s d)\end{array}$ & $20.1(0.6)$ & $20.01(0.61)$ & $20.07(0.50)$ & \\
\hline Waist circumference $(\mathrm{cm} ;$ mean $\pm \mathrm{sd})$ & $78.63( \pm 13.64)$ & $76.09( \pm 11.96)$ & $82.46( \pm 11.25)$ & $<0.001 \mathrm{a}<0.001 \mathrm{~b}<0.05 \mathrm{c}$ \\
\hline Body mass index (BMl, kg/m²) & $24.88(6.09)$ & $23.85(6.09)$ & $24.32(4.17)$ & $0.0504 c$ \\
\hline Glucose $(\mathrm{mmol} / \mathrm{L} ;$ mean $\pm \mathrm{sd})$ & $4.87( \pm 0.38)$ & $4.83( \pm 0.36)$ & $5.05( \pm 0.44)$ & $<0.001 \mathrm{a}<0.001 \mathrm{~b}$ \\
\hline Triglycerides (mmol/L;mean $\pm \mathrm{sd}$ ) & $0.85( \pm 0.38)$ & $1.16( \pm 0.49)$ & $1.08( \pm 0.56)$ & $<0.001 a<0.05 b<0.001 c$ \\
\hline $\mathrm{HDL}-\mathrm{C}(\mathrm{mmol} / \mathrm{L} ;$ mean $\pm \mathrm{sd})$ & $1.41( \pm 0.32)$ & $1.42( \pm 0.30)$ & $1.23( \pm 0.26)$ & $<0.001 \mathrm{a}<0.001 \mathrm{~b}$ \\
\hline $\mathrm{LDL}-\mathrm{C}(\mathrm{mmol} / \mathrm{L} ;$ mean $\pm \mathrm{sd})$ & $2.51( \pm 0.64)$ & $2.59( \pm 0.62)$ & $2.43( \pm 0.67)$ & $0.001 b$ \\
\hline $\begin{array}{l}\text { Systolic blood pressure } \\
(\mathrm{mm} \mathrm{Hg} \text {; mean } \pm \mathrm{sd})\end{array}$ & $110.92( \pm 11.69)$ & $111.7( \pm 10.65)$ & $123.2( \pm 12.4)$ & $<0.001 \mathrm{a}<0.001 \mathrm{~b}$ \\
\hline $\begin{array}{l}\text { Diastolic blood pressure } \\
(\mathrm{mm} \mathrm{Hg} ; \text { mean } \pm \mathrm{sd})\end{array}$ & $65.16( \pm 8.16)$ & $65.74( \pm 7.35)$ & $65.48( \pm 8.27)$ & \\
\hline Western diet (mean $\pm \mathrm{sd})$ & $-0.25( \pm 0.79)$ & $-0.4( \pm 0.68)$ & $0.41( \pm 0.94)$ & $<0.001 \mathrm{a}<0.001 \mathrm{~b}<0.05 c$ \\
\hline Healthy diet (mean \pm sd) & $0.01( \pm 0.84)$ & $-0.01( \pm 0.80)$ & $0.03( \pm 0.96)$ & \\
\hline Misreporting (n/\%) & & & & $<0.01 a<0.001 b$ \\
\hline Underreporting & $77(38.7)$ & $116(43.12)$ & $131(23.82)$ & \\
\hline Plausible reporting & $80(40.2)$ & $109(40.52)$ & $222(40.36)$ & \\
\hline Over-reporting & $8(4.02)$ & $11(4.09)$ & $33(6)$ & \\
\hline NA & $34(17.09)$ & $33(12.26)$ & $164(29.82)$ & \\
\hline Physical Activity (in the last 7 days, $n / \%$ ) & & & & $<0.001 \mathrm{a}<0.001 \mathrm{~b}$ \\
\hline Less than once & $39(20)$ & $55(20.45)$ & $36(6.55)$ & \\
\hline 1 to 3 times & $76(38.19)$ & $122(45.35)$ & $137(24.91)$ & \\
\hline More than 4 & $59(29.65)$ & $73(27.14)$ & $244(44.36)$ & \\
\hline NA & $25(12.56)$ & $19(7.06)$ & $133(24.18)$ & \\
\hline Sedentary behaviour (hours per day, $n / \%$ ) & & & & $<0.05 a<0.05 b$ \\
\hline 0 & / & $1(0.37)$ & / & \\
\hline 1 & $26(13.07)$ & $42(15.61)$ & $37(6.73)$ & \\
\hline 2 & $80(40.2)$ & $113(42.01)$ & $204(37.09)$ & \\
\hline 3 & $47(23.62)$ & $74(27.51)$ & $142(25.82)$ & \\
\hline 4 & $24(12.06)$ & $21(7.81)$ & $41(7.45)$ & \\
\hline NA & $22(11.06)$ & $18(6.69)$ & $126(22.91)$ & \\
\hline \multicolumn{5}{|l|}{ Smoking (currently, n/\%) } \\
\hline No & $154(77.39)$ & $217(80.67)$ & $355(64.55)$ & \\
\hline Yes & $23(11.56)$ & $33(12.27)$ & $68(12.36)$ & \\
\hline NA & $22(11.06)$ & $19(7.06)$ & $127(23.09)$ & \\
\hline $\begin{array}{l}\text { Alcohol consumption ((in the last } 7 \\
\text { days, } n / \% \text { ) }\end{array}$ & & & & $<0.001 a<0.01 c$ \\
\hline No & $103(51.76)$ & $110(40.89)$ & $158(28.37)$ & \\
\hline Yes & $70(35.18)$ & $137(50.93)$ & $258(46.91)$ & \\
\hline NA & $26(13.07)$ & $22(8.18)$ & $134(24.36)$ & \\
\hline \multicolumn{5}{|l|}{ Ethnicity $(n / \%)$} \\
\hline Caucasian & $159(79.9)$ & $234(86.99)$ & $452(82.18)$ & \\
\hline Not Caucasian & $34(17.09)$ & $33(12.27)$ & $84(15.27)$ & \\
\hline NA & $6(3.02)$ & $2(0.74)$ & $14(2.55)$ & \\
\hline
\end{tabular}




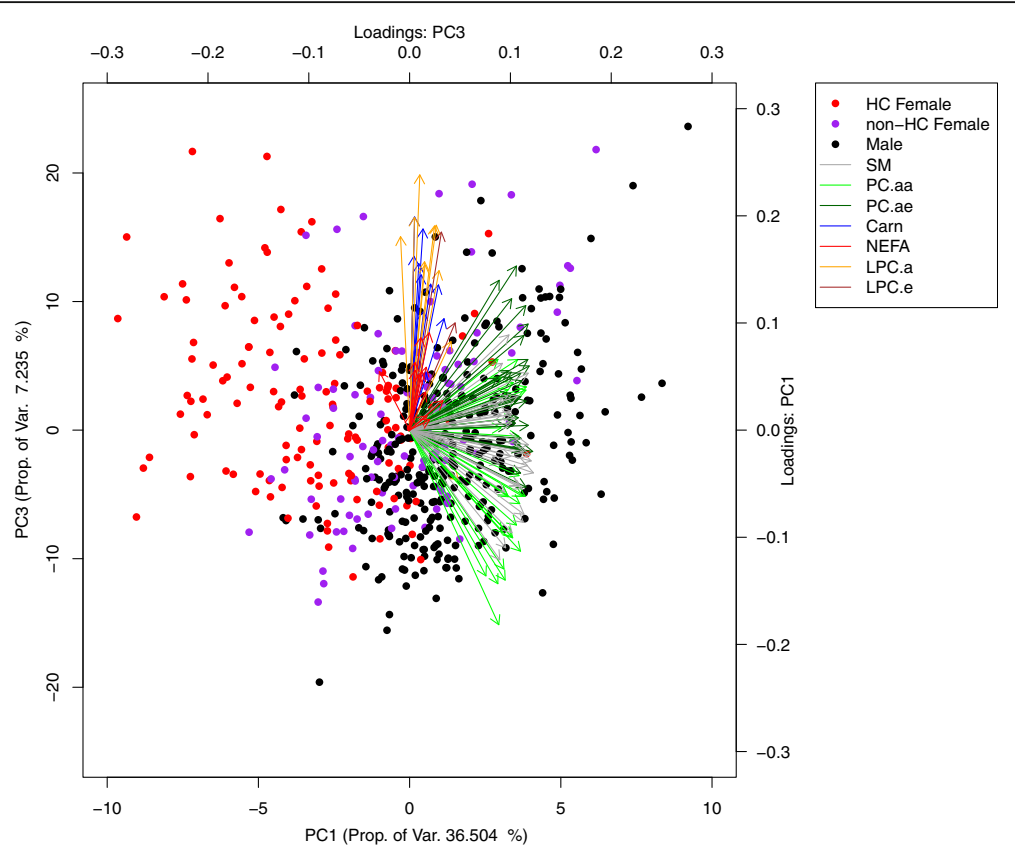

Fig. 2 Loadings plot of the first two components of the PCA. Arrows: metabolite groups that separate the data set into the different directions. Points: loading of the metabolite in the respective component according to male and $\mathrm{HC}$ female or non- $\mathrm{HC}$ female

For HDL, TAG and WC, LPCa C18:3 was significantly different. LPCa C18:1, LPCa C20:4, LPCa C22:6 and SMa C41:0 shared different associations depending on the sex category for HDL and WC.

HDL and TAG had significant differences in the metabolites LPCa C16:0, LPCa C18:0, LPCa C18:3, LPCe C18:1, PCaa C34:1, PCaa C34:3, PCaa C38:3, PCaa C38:4, SMa C36:0 and SMa C42:4 between the sex categories in common.

For HDL, LPCa C16:1, LPCa C20:3, LPCa C20:5, LPCa C22:5, LPCe C18:0, NEFA C24:5, PCaa C38:0, PCaa C38:6, PCaa C40:5, PCaa C40:6, PCae C40:0, PCae C40:6 and SMa C42:6 were significantly different between males, hormonal contraceptive-taking females and non-hormonal contraceptive-taking females.

TAG was significantly differently associated with PCaa C32:3, PCaa C36:0, PCaa C36:1, PCaa C36:2, PCaa C36:3, PCaa C36:4, PCaa C38:5, PCae C36:1, PCae C38:0, SMa C38:1, SMa C40:3 and SMa C40:4 (Fig. 3).

\section{Discussion}

Our results show significant differences in the concentrations of metabolites in males, $\mathrm{HC}$ and non- $\mathrm{HC}$ females. In general, males have higher LPC and Carn levels, and non-HC females have higher SM and PC levels. HC females have higher NEFA concentrations than non-HC females and males. The concentrations of HDL-C, glucose and TG, sysBP and WC differed significantly between males, $\mathrm{HC}$ and non-HC females and also in their associations with metabolite concentrations.
Numerous epidemiological studies have shown the prevalence of non-communicable diseases differs between males and females, and it is well established that sex hormones have a significant effect on certain diseases [ 1 , 4]. In the field of metabolomics, however, there are few studies addressing sex differences, and how they affect the metabolome underlying diseases risk factors $[3,15,16]$. Most of the metabolomics studies conducted to date have either not taken potential sex differences into account or have used males and females in the same model. There are several studies showing similar results as our study concerning the sex differences in metabolites [3, 15, 17]. However, to our knowledge there is only one other study that has examined sex differences with respect to $\mathrm{HC}$ use and

Table $\mathbf{2}$ Overview of number of significantly sex group between different metabolite groups

\begin{tabular}{llll}
\hline & Males vs hfemales & Males vs nhfemales & hfemales vs nhfemales \\
\hline AA & $15 \uparrow ; 1 \downarrow$ & $15 \uparrow ; 2 \downarrow$ & $4 \uparrow ; 12 \downarrow$ \\
Carn & $12 \uparrow$ & $11 \uparrow$ & $8 \downarrow$ \\
LPCa & $13 \uparrow$ & $11 \uparrow$ & $13 \downarrow$ \\
LPCe $\quad 3 \uparrow$ & $3 \uparrow$ & $3 \downarrow$ \\
NEFA & $2 \uparrow ; 16 \downarrow$ & $3 \uparrow ; 6 \downarrow$ & $6 \uparrow$ \\
PCaa & $32 \downarrow$ & $20 \downarrow$ & $20 \uparrow ; 2 \downarrow$ \\
PCae & $3 \uparrow ; 24 \downarrow$ & $1 \uparrow ; 20 \downarrow$ & $8 \uparrow ; 2 \downarrow$ \\
SM & $1 \uparrow ; 39 \downarrow$ & $1 \uparrow ; 35 \downarrow$ & $15 \uparrow ; 4 \downarrow$ \\
\hline
\end{tabular}

Arrows: $\uparrow$ meaning higher and $\downarrow$ meaning lower in the firstly mentiones group in the group comparison 


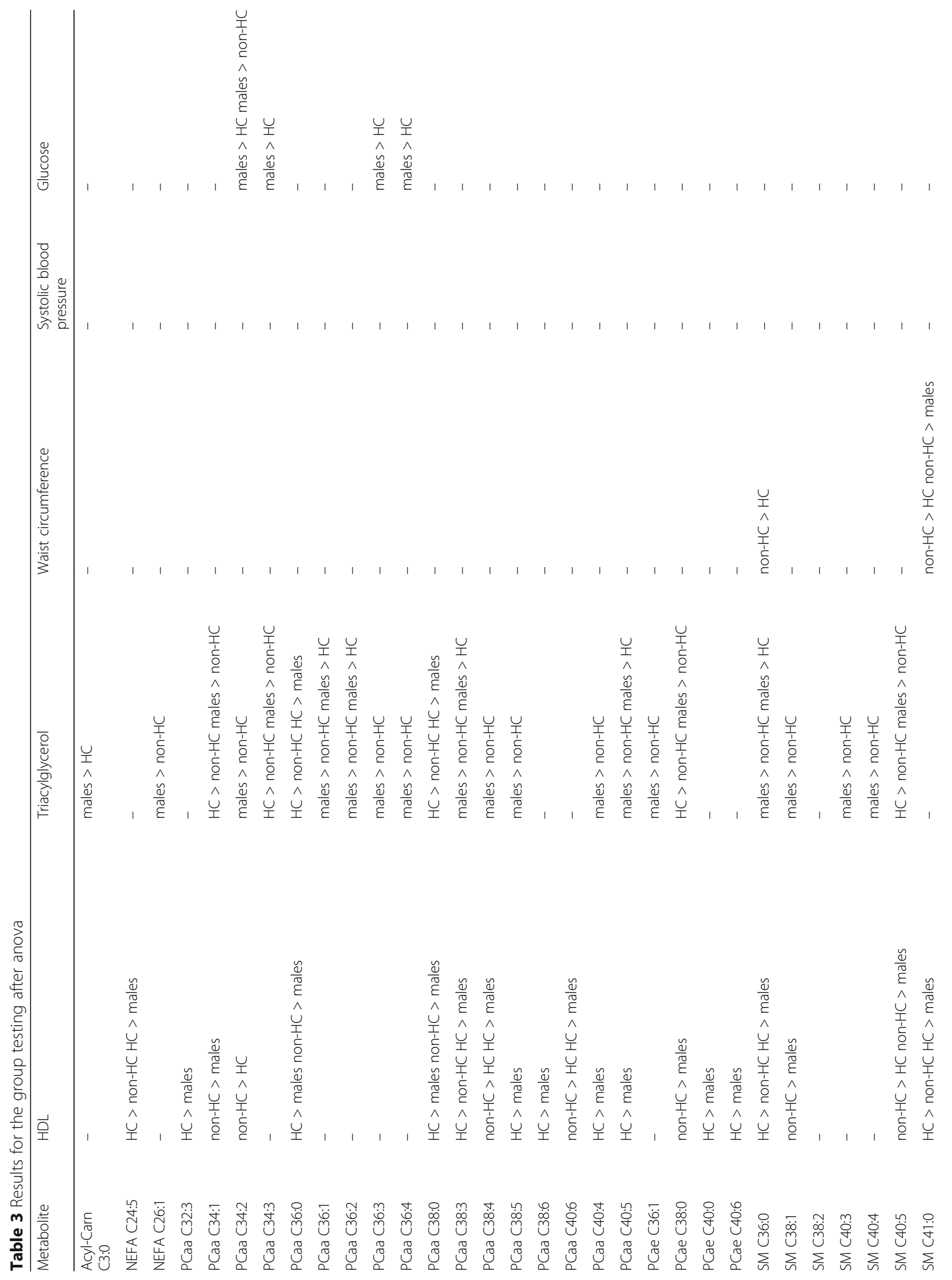


Rauschert et al. Biology of Sex Differences (2017) 8:10

Page 8 of 13

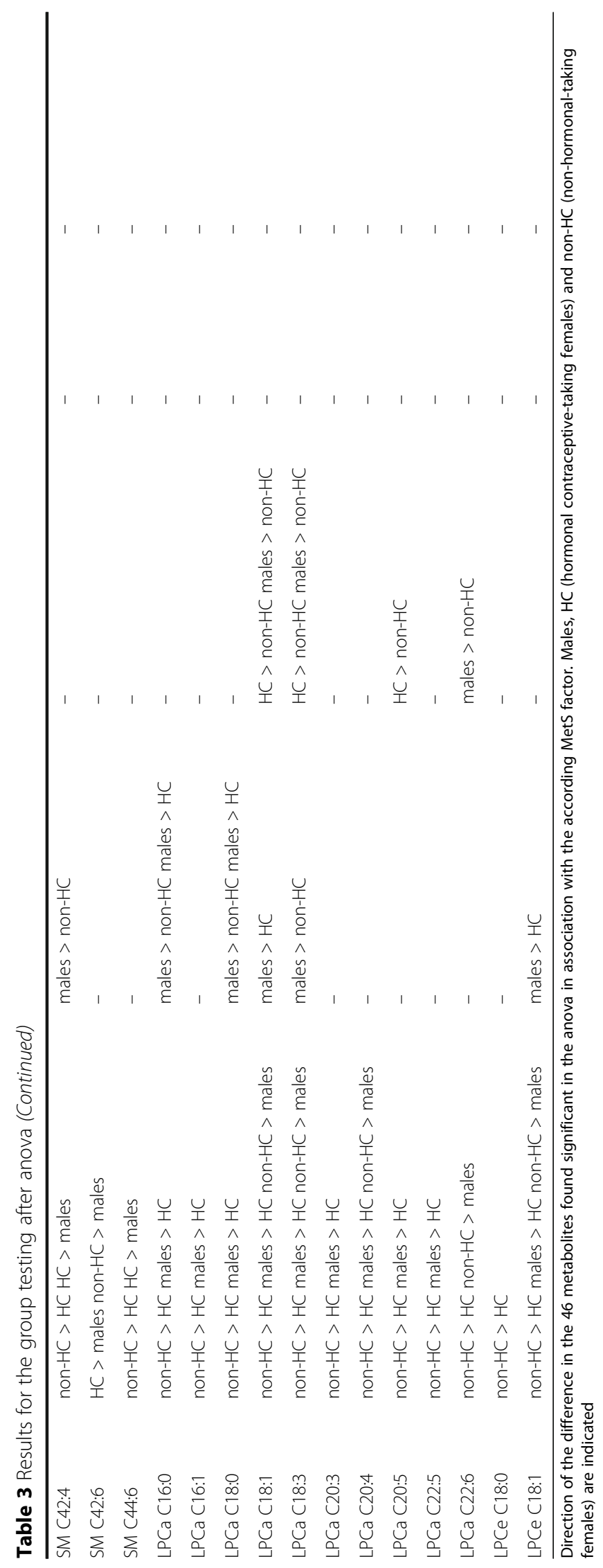




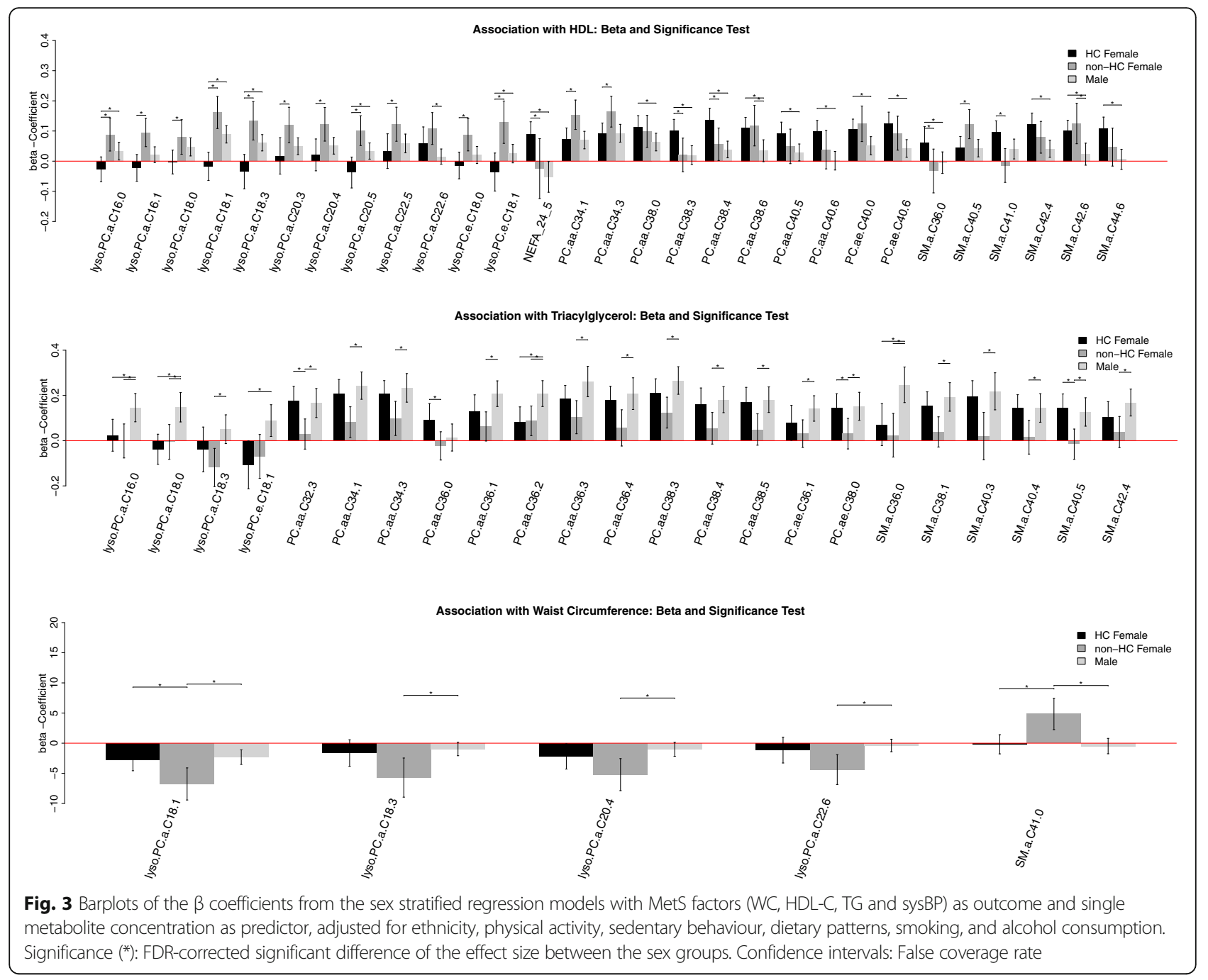

metabolomics or that has investigated associations between metabolomics with different components of the MetS [3, 15]. Given a significant number of women of childbearing age are taking $\mathrm{HC}$, this is a large group that is not represented or analysed in many clinical studies.

\section{General sex differences}

In the present study, we found higher WC in males than females which supports data showing obese males have more visceral adiposity than obese females [18]. Our data supports the literature in showing that HDL-C concentrations were higher in women than in men. Higher HDL-C is known to associate with lower cardiovascular risk [17]. Previous reports have shown that lower HDL-C in males associate with lower SM concentrations compared with that in females [19]. Our data are in accordance with these results.

We found LPC and SM concentrations most differentiating between males, non- $\mathrm{HC}$ and $\mathrm{HC}$ females. This might be due to a suppression of lecithin cholesterol acyltransferase (LCAT) activity by higher SM concentrations. Males, having lower SM concentrations than females, showed higher LPC concentrations, which is in accordance with LPCs being associated with higher LDL and lower HDL. The sex difference may also explain previous findings of decreased LPC concentrations in association with obesity, as LPC are higher in males than in females [20,21].

Although higher circulating LPC levels in the blood have been predominantly associated with LCAT activity [22], higher levels of LPC have also been associated with phospholipase $\mathrm{A}_{2}$ (PLA2) activity. This enzyme mainly associates with LDL and hydrolyses the fatty acid at the sn-2 position from phospholipids, leading to a LPC and a non-esterified fatty acid. This reaction by LPC by PLA2 is thought to play an important role in the early onset of cardiovascular diseases [23]. There is some evidence that PLA2 activity is increased in males compared with females, in accordance with our finding that LPCs were associated with male sex [24]. 
The difference in PC and SM levels between males and $\mathrm{HC}$ and non-HC females is likely related to the different distribution of lipoprotein species and their associated metabolic consequences. This may also explain some of the differences associated with HDL which is enriched in PC containing polyunsaturated fatty acids [25].

Serine was significantly higher in females. Together with palmitoyl-CoA concentrations, serine is the initial and rate-limiting metabolite in the metabolism of sphingolipids. We found that AA and especially branched chain amino acids (BCAA) concentrations were higher in males than in $\mathrm{HC}$ or non-HC females. BCAA and associated metabolites have been shown to associate with insulin resistance, cardiovascular disease and female sex hormones [26].

BCAA have been shown to be important in muscle metabolism, which could explain the significantly positive association of leucine with male sex rather than $\mathrm{HC}$ or non-HC females, since males have a higher percentage of lean body mass including muscle mass than females [27]. Testosterone is long known as an anabolic hormone linked to AA metabolism [28]. In a study in elderly men, testosterone supply led to increased strength and lean body weight [28]. In general the differences between males and females in the metabolome likely reflect differences in lipid and AA metabolism.

\section{Hormones and hormonal contraceptives}

The majority of $\mathrm{HC}$ provide the two hormones estrogen and progesterone, which influence AA and lipid metabolism [28-39]. We found that HC females have decreased free Carn and acyl-Carn levels together with decreased AA levels. Previous reports show female free Carn and acyl-Carn levels decrease significantly upon reaching fertile age [30]. It was suggested that estrogen levels might be the reason.

Furthermore, estrogen is associated with an increased availability of fatty acids by lipolysis and also decreases carbohydrate metabolism [19, 31].

A recent study showed that a decrease in estrogen enhances the accumulation of visceral fat in women through Aldehyde dehydrogenase 1 family, member A1 (aldh1a1), but the effect of the enzyme was not relevant in men, supporting sex differences in lipid metabolism driven by hormones [32].

Our observation of reduced concentrations of some NEFA species in males compared to non-HC females and especially $\mathrm{HC}$ females could be due to the higher testosterone levels in males [33]. Higher insulin concentrations are known to associate with lower NEFA levels that associate with a lower $\beta$-oxidation in the fasting state and therefore also lower acyl-Carn levels, as carrier of fatty acids.

Estrogen and estradiol have been shown to increase HDL-C blood concentrations, which is a protective marker for cardiovascular diseases. This could, in part, explain the increased risk of cardiovascular diseases in women after menopause [34]. In accordance with our findings, $\mathrm{HC}$ use has been associated with higher HDL-C and low-density lipoprotein-cholesterol (LDL-C) (higher than in males in our data), TG (lower in HC females compared to that in males and non-HC females in our data) concentrations [34]. This could explain the higher PC and $\mathrm{SM}$ concentrations in $\mathrm{HC}$ females compared to those in males and non-HC females, as they are the most abundant phospholipids in lipoproteins [35].

Some estrogen compounds have been shown to not only decrease concentrations of stearic acid but also, amongst others, increase palmitic acid in $\mathrm{PC}$ in the serum [38]. The lower levels of LPCa C16:0, LPCa C18:0 and LPCa C18:1 in $\mathrm{HC}$ females compared to non-HC females could be a reflection of that, since they potentially occur after cleaving a fatty acid from PCs by LCAT activity.

\section{MetS dependent differences}

Males in our study have a more adverse metabolic profile than non-HC females and $\mathrm{HC}$ females, with respect to $\mathrm{WC}$, sysBP and TG, HDL-C and glucose concentrations and also differences in the relationships between these components and different metabolites, for example LPC concentrations between males and either $\mathrm{HC}$ or non-HC females.

$\mathrm{HC}$ and non-HC females mainly differed in SM and PC concentrations. WC, TG and HDL were associated with increased LPC concentrations that are also differently elevated between males and $\mathrm{HC}$ and non- $\mathrm{HC}$ females. This is in keeping with the finding that LPC concentrations have been associated to WC in other studies and highlights the importance of our findings.

HDL levels are associated with LCAT, an enzyme that leads to the formation of LPC [36]. In our study, males had higher levels of LPC and lower levels of HDL-C than females.

HDL is known to contain a lower SM/PC ratio than LDL, which could be a reason for HDL being mainly associated with PC and LPC species in our study [37]. The $\beta$ coefficient for the association between LPC levels and components of the MetS was higher in females than in males; irrespective of whether it was a positive (sysBP, TG, HDL) or negative (TG, WC, HDL) association. This suggests a more negative effect of LPC in females than in males, possibly due to the fact, that males already have higher levels of LPC.

In our study SM had a strong association with high levels of components of the MetS in males and in $\mathrm{HC}$ or non-HC females. But in general, SM concentrations were higher in females ( $\mathrm{HC}$ and non-HC) than in males.

To our knowledge there are no other studies that have performed a comprehensive metabolomics analysis examining sex differences in relation to components of the 
MetS. Newbern et al. reported sex differences in insulin resistance in adults in their metabolomics study, although these were mainly in BCAAs and their by-products shortchain acyl-Carn [15].

Because of the young age of 20 years of the participants, the analysis of this cohort is valuable for future lifestyle and other prevention strategies in this age group. Additionally, the sample size is exceptionally high with more than 1000 participants. Studies like ours provide some insight into the possible mechanisms for the differences in the prevalence of diseases between sexes. These data are also important in assisting intervention and metabolomics studies conducted in the future.

The limitations of our study are that we do not have blood sex hormone concentrations or any information on the time of the estrous cycle, so the focus of this study is hypothesis generation. It is an observational cohort study with a cross-sectional analysis, which does not allow for interpretations of the directions of associations. Additionally, we had only plasma and no cell samples to measure and interpret metabolic changes, and we did not have separated lipoprotein species.

\section{Conclusions}

This study has shown clear differences between plasma metabolite concentrations in males and $\mathrm{HC}$ or non- $\mathrm{HC}$ females, especially in LPC, SM and PC, which have been shown to associate with obesity in other studies $[7,21,38]$. Further, the association of these metabolites differed between sexes with components of the MetS, which means that development of diseases like obesity and diabetes may differ between the sexes, potentially mediated by sex hormones. Our findings highlight the importance of considering sex differences when conducting a metabolomics study, and the need to account for the effect of HC usage in females in future studies. The latter finding could be of importance for early programming or pregnancy studies, as the use of $\mathrm{HC}$ leads to a hormonal state in the body similar to pregnant women $[29,39]$. To our knowledge this is the first comprehensive analysis demonstrating differences in metabolomics between males and females, as well as the effect of $\mathrm{HC}$ use. Additionally, we show that the association between metabolomics markers and components of the MetS are significantly different between males and females.

\section{Additional file}

Supplemental information is available on the website of Biology of Sex Differences.

Additional file 1: Table S1. Results for the regression model of the unstratified population with the five MetS. Table S2-S4. Results of the Regression model with Metabolite concentration as outcome and two-level sex variable as predictor. Table S5. Results for the ANOVA for the five MetS Factors. Table S6: Results for the group testing after the
Anova. Table S6.1. Results for the testing of hfemales versus nhfemales. Table 6.2. Results for the testing of males versus hfemales. Table S6.3. Results for the testing of males versus nhfemales. Table 7. Median, 25\% and $75 \%$ quartile for every metabolite (215) of the Raine Study metabolomics dataset stratified by males and non-hormonal and hormonal contraceptivetaking females. Table S7.1. Non-hormonal contraceptive-taking females. Table S7.2. Hormonal contraceptive-taking females. Table S7.3. Male subset. (DOCX $530 \mathrm{~kb}$ )

\section{Abbreviations}

AA: Amino acids; acyl-Carn: Acyl-Carnitine; aldh1a1: Aldehyde dehydrogenase 1 family, member A1; Anova: Analysis of variance; BCAA: Branched chain amino acids; Carn: Free carnitine; CoA: Coenzyme A; HC females: Hormonal contraceptive-taking females; HDL-C: High-density lipoprotein cholesterol; LCAT: Lecithin cholesterol acyltransferase; LC-MS/MS: Liquid chromatographytandem mass spectrometry; LDL-C: Low-density lipoprotein cholesterol; LPC/ LPCa: Lyso-phosphatidylcholines; LPCe: alkyl-linked lyso-phosphatidylcholines; MetS: Metabolic syndrome; NEFA: Non-esterified fatty acids; Non-HC females: Non-hormonal contraceptive-taking females; PCA: Principal component analysis; PCaa: Diacyl-phosphatidylcholines; PCae: Acyl-alkyl-phosphatidylcholines; SM: Sphingomyelines; sysBP: Systolic blood pressure; TG: Triacylglycerol; WC: Waist circumference

\section{Acknowledgements}

We are grateful to Stephanie Winterstetter (Division of Metabolic and Nutritional Medicine, Dr von Hauner Children's Hospital, University of Munich, Munich, Germany), who analysed the blood plasma samples, and to Franca Kirchberg, for statistical advices.

The data presented are part of the PhD thesis accomplished by Sebastian Rauschert at the Medical Faculty of the Ludwig-Maximilians-University of Munich. Also, we gratefully acknowledge the Raine Study participants and their families, and the Raine Study Team, for the cohort coordination and data collection.

\section{Funding}

Core funding for the Western Australian Pregnancy Cohort (Raine) Study is provided by the University of Western Australia; the Faculty of Medicine, Dentistry and Health Sciences at the University of Western Australia; the Telethon Kids Institute (formerly known as the Telethon Institute for Child Health Research); the Women and Infants Research Foundation; Curtin University of Technology; Edith Cowan University and the Raine Medical Research Foundation. We thank the National Health and Medical Research Council (NHMRC) for their provision of funding (Project ID\#1022134 and European Union collaborative project ID\#1037966). TM is supported by an NHMRC Research Fellowship (1042255).

Further funding was received from the European Union's 7th Framework Programme (FP7/2007-2013), EarlyNutrition project, under grant agreement No. 289346, and from the European Research Council Advanced Grant ERC-2012-AdG - no.322605 META-GROWTH.

This manuscript does not necessarily reflect the views of the Commission of the European Communities and in no way anticipates the future policy in this area. The supporters of this work had no role in the study design, the data collection and analysis, the decision to publish, or the preparation of the manuscript.

\section{Availability of data and materials}

The datasets during and/or analysed during the current study are available from the corresponding author on reasonable request.

\section{Authors' contributions}

SR wrote the paper and performed the statistical analysis and data interpretation. $\mathrm{CH}$ contributed to the statistical analysis and data interpretation. OU performed LC-MS/MS analysis and contributed to data interpretation. BK and WO conceived the study. TM was responsible for the collection, storage and shipment of plasma to Germany, for metabolomics analysis. TM, LB and WO were responsible for all data collection. All co-authors have contributed to the content, read and approved the final manuscript. 


\section{Competing interests}

The authors declare that they have no competing interests.

\section{Consent for publication}

Not applicable.

\section{Ethics approval and consent to participate}

Ethics approval at the 20-year follow-up was obtained from the University of Western Australia Human Research Ethics Committee. Informed and written consent was obtained from participants.

\section{Publisher's Note}

Springer Nature remains neutral with regard to jurisdictional claims in published maps and institutional affiliations.

\section{Author details}

'Ludwig-Maximilian Universität München, Dr. von Hauner Children's Hospital, University of Munich Medical Center, Lindwurmstr. 4, D-80337 München, Germany. ${ }^{2}$ School of Medicine and Pharmacology, Royal Perth Hospital Unit, University of Western Australia, Perth, Western Australia 6000, Australia. ${ }^{3}$ Menzies Institute for Medical Research, University of Tasmania, Hobart, Tasmania 7000, Australia. ${ }^{4}$ Telethon Kids institute, The University of Western Australia, Perth, Western Australia 6009, Australia.

\section{Received: 6 October 2016 Accepted: 14 March 2017}

Published online: 28 March 2017

\section{References}

1. Leening MJ, Ferket BS, Steyerberg EW, Kavousi M, Deckers JW, Nieboer D, et al. Sex differences in lifetime risk and first manifestation of cardiovascular disease: prospective population based cohort study. BMJ. 2014;349:95992. Pubmed Central PMCID: PMC4233917. Epub 2014/11/19. eng.

2. Morrow EH. The evolution of sex differences in disease. Biol Sex Differ. 2015; 6:5. Pubmed Central PMCID: PMC4359385, Epub 2015/03/17. eng.

3. Ruoppolo M, Campesi I, Scolamiero E, Pecce R, Caterino M, Cherchi S, et al. Serum metabolomic profiles suggest influence of sex and oral contraceptive use. Am J Transl Res. 2014;6(5):614-24. Pubmed Central PMCID: PMC4212935, Epub 2014/11/02. eng.

4. Bassuk SS, Manson JE. Oral contraceptives and menopausal hormone therapy: relative and attributable risks of cardiovascular disease, cancer, and other health outcomes. Ann Epidemiol. 2015;25(3):193-200.

5. Kanter R, Caballero B. Global gender disparities in obesity: a review. Adv Nutr. 2012;3(4):491-8. Pubmed Central PMCID: PMC3649717. Epub 2012/07/17. eng.

6. Alberti KG, Zimmet P, Shaw J. Metabolic syndrome-a new world-wide definition. A Consensus Statement from the International Diabetes Federation. Diabet Med. 2006;23(5):469-80. Epub 2006/05/10. eng.

7. Rauschert S, Uhl O, Koletzko B, Kirchberg F, Mori TA, Huang RC, et al. Lipidomics reveals associations of phospholipids with obesity and insulin resistance in young adults. J Clin Endocrinol Metab. 2015:jc20153525. Epub 2015/12/29. Eng.

8. Newnham JP, Evans SF, Michael CA, Stanley FJ, Landau LI. Effects of frequent ultrasound during pregnancy: a randomised controlled trial. Lancet. 1993;342(8876):887-91. Epub 1993/10/09. eng.

9. Hellmuth C, Weber M, Koletzko B, Peissner W. Nonesterified fatty acid determination for functional lipidomics: comprehensive ultrahigh performance liquid chromatography-tandem mass spectrometry quantitation, qualification, and parameter prediction. Anal Chem. 2012;84(3): 1483-90. Epub 2012/01/10. eng.

10. Harder U, Koletzko B, Peissner W. Quantification of 22 plasma amino acids combining derivatization and ion-pair LC-MS/MS. J Chromatogr B Anal Technol Biomed Life Sci. 2011;879(7-8):495-504. Epub 2011/02/05. eng.

11. Ambrosini GL, Oddy WH, Robinson M, O'Sullivan TA, Hands BP, de Klerk NH, et al. Adolescent dietary patterns are associated with lifestyle and family psychosocial factors. Public Health Nutr. 2009;12(10):1807-15. Epub 2009/01/24. eng.

12. Fox J, Weisberg S. An $\{R\}$ Companion to Applied Regression. Secondth ed. Thousand Oaks: Sage; 2011. URL: http://socserv.socsci.mcmaster.ca/jfox/ Books/Companion.

13. Benjamini $Y$, Hochberg Y. Controlling the false discovery rate: a practical and powerful approach to multiple testing. J R Stat Soc B Methodol. 1995; 57(1):289-300.
14. Benjamini Y, Yekutieli D. False discovery rate-adjusted multiple confidence intervals for selected parameters. J Am Stat Assoc. 2005; 100(469):71-81.

15. Newbern D, Gumus Balikcioglu P, Balikcioglu M, Bain J, Muehlbauer M, Stevens $R$, et al. Sex differences in biomarkers associated with insulin resistance in obese adolescents: metabolomic profiling and principal components analysis. J Clin Endocrinol Metab. 2014;99(12):4730-9. Pubmed Central PMCID: PMC4328030, Epub 2014/09/10. eng.

16. Won EY, Yoon MK, Kim SW, Jung Y, Bae HW, Lee D, et al. Gender-specific metabolomic profiling of obesity in leptin-deficient ob/ob mice by $1 \mathrm{H}$ NMR spectroscopy. PLoS One. 2013;8(10):e75998. Pubmed Central PMCID: PMC3789719, Epub 2013/10/08. eng.

17. Mittelstrass K, Ried JS, Yu Z, Krumsiek J, Gieger C, Prehn C, et al. Discovery of sexual dimorphisms in metabolic and genetic biomarkers. PLOS Genet. 2011; 7(8):e1002215. Pubmed Central PMCID: 3154959.

18. Karastergiou K, Smith SR, Greenberg AS, Fried SK. Sex differences in human adipose tissues - the biology of pear shape. Biol Sex Differ. 2012;3(1):13. Pubmed Central PMCID: PMC3411490, Epub 2012/06/02. eng.

19. Wu BN, O'Sullivan AJ. Sex differences in energy metabolism need to be considered with lifestyle modifications in humans. J Nutr Metab. 2011;2011: 391809. Pubmed Central PMCID: PMC3136178, Epub 2011/07/21. eng.

20. Reinehr T, Wolters B, Knop C, Lass N, Hellmuth C, Harder U, et al. Changes in the serum metabolite profile in obese children with weight loss. Eur J Nutr. 2015;54(2):173-81. Epub 2014/04/18. eng.

21. Barber MN, Risis S, Yang C, Meikle PJ, Staples M, Febbraio MA, Bruce CR. Plasma lysophosphatidylcholine levels are reduced in obesity and type 2 diabetes. PLoS One. 2012;7(7):e41456. Pubmed Central PMCID: Pmc3405068, Epub 2012/08/01. eng.

22. Matsumoto T, Kobayashi T, Kamata K. Role of lysophosphatidylcholine (LPC) in atherosclerosis. Curr Med Chem. 2007;14(30):3209-20.

23. Lavi S, McConnell JP, Rihal CS, Prasad A, Mathew V, Lerman LO, et al. Local production of lipoprotein-associated phospholipase A2 and lysophosphatidylcholine in the coronary circulation association with early coronary atherosclerosis and endothelial dysfunction in humans. Circulation. 2007;115(21):2715-21.

24. Brilakis ES, Khera A, McGuire DK, See R, Banerjee S, Murphy SA, et al. Influence of race and sex on lipoprotein-associated phospholipase A2 levels: observations from the Dallas Heart Study. Atherosclerosis. 2008; 199(1):110-5

25. Kontush A, Lhomme M, Chapman MJ. Unraveling the complexities of the HDL lipidome. J Lipid Res. 2013;54(11):2950-63. Pubmed Central PMCID: PMC3793600, Epub 2013/04/02. eng.

26. Lamont LS, McCullough AJ, Kalhan SC. Gender differences in the regulation of amino acid metabolism. J Appl Physiol. 2003;95(3):1259-65. Epub 2003/06/17. eng.

27. Janssen I, Heymsfield SB, Wang ZM, Ross R. Skeletal muscle mass and distribution in 468 men and women aged 18-88 yr. J Appl Physiol. 2000; 89(1):81-8. Epub 2000/07/25. eng.

28. Mauras N, Hayes V, Welch S, Rini A, Helgeson K, Dokler M, Veldhuis JD, Urban RJ. Testosterone deficiency in young men: marked alterations in whole body protein kinetics, strength, and adiposity. J Clin Endocrinol Metab. 1998;83(6):1886-92. Epub 1998/06/17. eng.

29. Spona J, Dusterberg B, Ludicke F. Composition for contraception. Google Patents. 2013.

30. Rasmussen J, Nielsen OW, Janzen N, Duno M, Gislason H, Kober L, et al. Carnitine levels in 26,462 individuals from the nationwide screening program for primary carnitine deficiency in the Faroe Islands. J Inherit Metab Dis. 2014;37(2):215-22. Epub 2013/05/09. eng.

31. D'Eon T, Braun B. The roles of estrogen and progesterone in regulating carbohydrate and fat utilization at rest and during exercise. J Womens Health Gend Based Med. 2002;11(3):225-37. Epub 2002/05/04. eng.

32. Yasmeen R, Reichert B, Deiuliis J, Yang F, Lynch A, Meyers J, et al. Autocrine function of aldehyde dehydrogenase 1 as a determinant of diet- and sexspecific differences in visceral adiposity. Diabetes. 2013:62(1):124-36. Pubmed Central PMCID: PMC3526050, Epub 2012/08/31. eng.

33. Laaksonen DE, Niskanen L, Punnonen K, Nyyssonen K, Tuomainen TP, Salonen R, et al. Sex hormones, inflammation and the metabolic syndrome: a population-based study. Eur J Endocrinol. 2003;149(6):601-8. Epub 2003/12/04. eng.

34. Naz F, Jyoti S, Akhtar N, Afzal M, Siddique YH. Lipid profile of women using oral contraceptive pills. PJBS. 2012;15(19):947-50. Epub 2013/10/29. eng. 
35. Sultan N, Nawaz M, Sultan A, Fayaz M, Baseer A. Effect of menopause on serum HDL-cholesterol level. JAMC. 2003;15(3):24-6. Epub 2004/01/20. eng.

36. Subbaiah PV, Liu M. Role of sphingomyelin in the regulation of cholesterol esterification in the plasma lipoproteins. Inhibition of lecithin-cholesterol acyltransferase reaction. J Biol Chem. 1993;268(27): 20156-63. Epub 1993/09/25. eng.

37. Wiesner P, Leidl K, Boettcher A, Schmitz G, Liebisch G. Lipid profiling of FPLC-separated lipoprotein fractions by electrospray ionization tandem mass spectrometry. J Lipid Res. 2009;50(3):574-85. Epub 2008/10/04. eng.

38. Floegel A, Stefan N, Yu Z, Muhlenbruch K, Drogan D, Joost HG, et al. Identification of serum metabolites associated with risk of type 2 diabetes using a targeted metabolomic approach. Diabetes. 2013;62(2):639-48. Pubmed Central PMCID: PMC3554384, Epub 2012/10/09. eng.

39. Yannone ME. Hormonal changes in pregnancy. MCV/Q. 1972;8(1):43-51.

Submit your next manuscript to BioMed Central and we will help you at every step:

- We accept pre-submission inquiries

- Our selector tool helps you to find the most relevant journal

- We provide round the clock customer support

- Convenient online submission

- Thorough peer review

- Inclusion in PubMed and all major indexing services

- Maximum visibility for your research

Submit your manuscript at www.biomedcentral.com/submit
Biomed Central 